\title{
ELENCANDO CUIDADOS DE ENFERMAGEM AO PACIENTE ETILISTA CRÔNICO E PORTADOR DE TUBERCULOSE PULMONAR: UM RELATO DE CASO
}

Débhora Ísis Barbosa e SILVA ${ }^{1}$

Felipe Artur Gomes de ASSIS²

\begin{abstract}
${ }^{1}$ Enfermeira. Especialista em Saúde da Família. Residente de Enfermagem em Pneumologia. Email: debhoraisis@gmail.com

${ }^{2}$ Enfermeiro. Especialista em Saúde da Família. Residente de Enfermagem em Pneumologia. Email: euartur01@gmail.com
\end{abstract}

Recebido em: 25/03/2015 - Aprovado em: 15/08/2015 - Disponibilizado em: 30/10/2015

\begin{abstract}
RESUMO: A tuberculose é uma doença infecto-contagiosa, causada por um microorganismo denominado Mycobacterium tuberculosis, também denominado de bacilo de Koch (BK). Apresentando evolução em ciclos lentos e de maior incidência nas aglomerações urbanas, transmitida através do ar, por meio de gotículas contendo os bacilos expelidos pelo doente ao tossir, espirrar ou falar em voz alta. É uma doença curável. Este relato tem como objetivo a realização de uma análise comparativa entre as informações disponíveis na literatura acerca da Tuberculose e Etilismo frente aos sinais e sintomas encontrados no paciente em estudo, descrever os cuidados de enfermagem e a evolução clínica esperada do referido paciente. Realizou-se acompanhamento do paciente durante o período de internação, 01 a 15 de setembro de 2014, em um serviço hospitalar de nível terciário da Região Metropolitana do Recife. Os dados foram obtidos por meio de entrevista, resgate histórico da avaliação do serviço social, exame fisco e análise do prontuário contextualizando e agrupando informações a fim de proporcionar lista de cuidados de enfermagem. Os cuidados prestados pela equipe de enfermagem do setor de Tisiologia com a colaboração dos Residentes de Enfermagem em Pneumologia obteve como resultado a melhora substancial promovendo a alta hospitalar do paciente após três meses de internamento.

Palavras chave: Tuberculose. Tuberculose Pulmonar. Cuidados de Enfermagem. Assistência Integral à Saúde. Planejamento de Assistência ao Paciente.
\end{abstract}

\section{ELENCANDO TO NURSING CARE PATIENT AND CHRONIC alcoholic CARRIER PULMONARY TUBERCULOSIS: A CASE REPORT}

\begin{abstract}
Tuberculosis is an infectious disease cause by a microorganism called Mycobacterium tuberculosis, it is also called bacillus of Koch (BK). It shows development at low cycle rates and it's higher incidence is in the urban areas, it is transmitted through the air, through drops of saliva containing the bacillus when a patient coughs, sneezes or speaks out loud. It is a curable disease. This report aims at carrying out a comparative analysis between the available literature on tuberculosis and alcohol consumption taking into consideration the signs and symptoms seen in the patient of the study, describing nursing care and the expected clinical evolution of the referred patient. A follow-up was carried out during the time the patient was hospitalized, from the $1^{\text {st }}$ to $15^{\text {th }}$ of September, in a tertiary level hospital in the metropolitan area of Recife. The data was obtained through interviews, retrieval of social service evaluation, physical examination and medical record analysis and all the pieces of information were contextualized and grouped in order to make a list of nursing care procedures. Nursing care provided by the Thoracic department in cooperation with the Pneumology Nursing Residency team obtained considerable improvement in the patient's condition leading to hospital discharge after three months of hospitalization.

Keywords: Tuberculosis. Pulmonary tuberculosis. Nursing Care. Comprehensive Health Care. Patient Care Planning.
\end{abstract}




\section{CONSIDERAÇÕES INICIAIS}

A tuberculose é uma doença infecto-contagiosa, causada por um microorganismo denominado Mycobacterium tuberculosis, também denominado de bacilo de Koch (BK). Apresentando evolução em ciclos lentos e de maior incidência nas aglomerações urbanas, transmitida através do ar, por meio de gotículas contendo os bacilos expelidos pelo doente ao tossir, espirrar ou falar em voz alta. Quando pessoas sadias entram em contato com pessoas que apresentam o bacilo de Kock, elas provocam a infecção e o risco de desenvolver a doença. É uma doença curável (Ministério da Saúde, 2002).

Anualmente são notificados cerca de 6 milhões de novos casos em todo o mundo, levando mais de um milhão de pessoas a óbito, compondo um problema de saúde prioritário no Brasil, que juntamente com outros 21 países em desenvolvimento, albergam $80 \%$ dos casos mundiais da doença. No Brasil, a tuberculose é sério problema da saúde pública, com profundas raízes sociais. A cada ano, são notificados aproximadamente 70 mil casos novos e ocorrem 4,6 mil mortes em decorrência da doença. O Brasil ocupa o $17^{\circ}$ lugar entre os 22 países responsáveis por $80 \%$ do total de casos de tuberculose no mundo. (Ministério da Saúde, 2005).
Para que esse quadro seja revertido os profissionais de saúde, principalmente os que atuam na Estratégia de Saúde da Família (ESF) - porta de entrada do SUS - precisam estar atentos e capacitados para realizar o pronto diagnóstico dos casos suspeitos, iniciar rapidamente o tratamento e acompanhar os pacientes, visando à cura plena (SLVA et al., 2011)

No Brasil, os medicamentos usados nos esquemas padronizados para a tuberculose são a isoniazida (H), a rifampicina (R), a pirazinamida (Z) e o etambutol (E). A maior parte das pessoas são tratadas pelos esquemas padronizados e receberá o tratamento e acompanhamento na atenção básica (Ministério da Saúde, 2002).

Atualmente, o percentual de cura da tuberculose não ultrapassa $75 \%$ dos casos tratados, embora o Brasil tenha sido o primeiro País a implantar o tratamento de curta duração - seis meses - em 1980, obtendo relativo sucesso inicial. O percentual insatisfatório de cura decorre, sobretudo, do abandono do tratamento que, logo no início, confere ao paciente uma melhora notável. A reversão desse quadro depende, principalmente, dos profissionais de saúde. Eles precisam estar atentos e devidamente capacitados para informar a população acerca da doença e dos meios de preveni-la, bem como para realizar o pronto diagnóstico dos casos suspeitos, iniciarem rapidamente o 
tratamento e acompanhar os pacientes, de modo a garantir-lhes a cura plena (Ministério da Saúde, 2002).

Em 2007, o Governo Federal lançou a Política Nacional sobre o Álcool (PNA), trazendo um conjunto de medidas para reduzir e prevenir os danos à saúde e à vida, apresentando princípios fundamentais à sustentação de estratégias, para o enfrentamento coletivo dos problemas relacionados ao consumo de álcool (SEGATTO; PINSKY; LARANJEIRA; REZENDE; VILELA; 2007)

O alcoolismo exerce influência sobre o prognóstico e o tratamento da tuberculose (TB), visto que há alta incidência de casos e de formas mais avançadas de TB pulmonar entre pacientes alcoolistas. $\mathrm{O}$ problema deve ser tratado na comunidade e, também, mais considerado pela equipe de saúde que trabalha diretamente com doentes de TB, buscando encontrar meios precisos de identificar esses pacientes e oferecer tratamento concomitante ao uso ou abuso de álcool. (ANDRADE; VILLA; PILLON, 2005)

De acordo com Andrade, Villa e Pillon (2005), os alcoolistas apresentaram probabilidade quase quatro vezes maior de abandonar o tratamento. A sensação de recuperação de saúde, o uso da bebida alcoólica ou drogas e o desconforto do tratamento - incluindo efeitos adversos - são motivos para o abandono do tratamento, segundo a percepção de alguns pacientes. Detectar o consumo de álcool, ou sua dependência, durante o tratamento da TB, é importante para evitar possíveis complicações ao usuário do Programa de Controle da Tuberculose - PCT.

Segundo Albuquerque, Leitão, Campelo, Souza e Salustiano (2001), um dos principais problemas encontrados pelos pacientes é a não adesão ao tratamento oferecido, tornando-se paciente crônico, tanto da doença, quanto do serviço. Verifica-se que entre os usuários de álcool há maior cooperação desses em relação ao tratamento quando submetidos a vigilância maior e, por isso, é indicado para tal clientela o Tratamento Diretamente Observado (DOTS) da TB, que determina maior adesão ao tratamento e consequientemente maior taxa de cura e menor desenvolvimento de resistência aos medicamentos.

\section{OBJETIVO}

Este relato tem como objetivo a realização de uma análise comparativa entre as informações disponíveis na literatura acerca da Tuberculose e Etilismo frente aos sinais e sintomas encontrados no paciente em 
estudo, descrever os cuidados de enfermagem e a evolução clínica esperada do referido paciente.

\section{MÉTODO}

Realizou-se acompanhamento do paciente durante o período de internação, 01 a 15 de setembro de 2014, em um serviço hospitalar de nível terciário da Região Metropolitana do Recife. Os dados foram obtidos por meio de entrevista, resgate histórico da avaliação do serviço social, exame fisco e análise do prontuário contextualizando e agrupando informações a fim de proporcionar lista de cuidados de enfermagem.

\section{RELATO DE CASO}

Paciente do sexo masculino, 47 anos, morador de rua, não recorda informações relevantes quanto sua história socioeconômica e cultural, foi admitido em um hospital de grande porte do Recife após ser transferido de uma Unidade de Pronto Atendimento da Região Metropolitana do Recife, com história de crise convulsiva não presenciada sendo traçado o diagnóstico médico inicial de tuberculose pulmonar evidenciada através de imagens radiográficas sugestivas de cavitações em virtude do infiltrado heterogêneo bilateral com áreas de cavidade.

Internado na Emergência clínica em meados de julho de 2014, sendo transferido posteriormente para o setor da Tisiologia no mesmo hospital.

Ao avaliar o padrão de atividade funcional juntamente ao exame físico, constatamos a total dependência da equipe de enfermagem para o desenvolvimento do cuidado para comer, beber, vestir-se, mobilidade no leito e ingestão dos medicamentos prescritos.

Em seu padrão cognitivo mostrouse consciente, embora desorientado quanto ao tempo e espaço, apresentava-se sonolento. Com a fala arrastada e murmurada, demonstrou pouca habilidade de compreensão dos questionamentos realizados e demonstrando audição e visão preservadas.

Com a silhueta bastante emagrecida e o apetite aumentado não percebemos alteração na sensação do paladar nem dos hábitos intestinais, não obstante a ausência da prótese total superior não interferiu nesse processo. Sendo um individuo longilíneo de grande porte que enfrentou um período prolongado na Emergência Clínica da unidade hospitalar, onde foi realizado um Cateterismo Vesical de Demora para coleta da urina sem evidencia de incontinência, sendo o volume adequado para a idade e hidratação hídrica realizada. 
A pele em região sacra apresentou-se descontínua pela presença de uma úlcera por pressão de Grau III medindo aproximadamente $7 \mathrm{~cm}$ de diâmetro, $5 \mathrm{~cm}$ de largura e $6 \mathrm{~mm}$ de profundidade com bordas delimitadas, com presença significativa de esfacelos sem visualização de tecido ósseo.

Houve registro da presença de febre matinal persistente sem alteração da freqüência cardíaca e valores da pressão arterial, sendo necessária a correção medicamentosa, contudo, a freqüência respiratória de aspecto dispnéico lento sem monitoração adequada foi presenciada.

O exame de baciloscopia confirmou a presença do bacilo de Kock em três cruzes, realizado após quinze dias do internamento através do método de coloração de Ziehl-Neelsen, tal retardo na realização se deu em virtude do quadro clínico comprometido, a desorientação, a confusão mental e a pouca contactuação do paciente com os interlocutores, impossibilitando desta forma a coleta de material adequado para realização deste exame.

Cinco dias, após internamento no setor da Tisiologia, apresentou sintomas característico da síndrome abstinência alcoólica de forma intensificada, como o aumento da desorientação associado a tremores de membros e agitação motora e ainda a desidratação. A presença da tosse produtiva foi constatada deste a primeira avaliação médica na emergência, onde a dificuldade na expectoração elencada como um ponto de risco obteve melhora após aumento do nível de consciência indicando resposta reflexa involuntária.

Dentre os exames

complementares solicitados estão a Tomografia Computadorizada de Crânio com resultado sem anormalidades evidentes [não há formações expansivas ou sangramentos intraparenquimatosos; não há desvios das estruturas da linha média ou coleções extraaxiais]; exames bioquímicos e hematológicos sem alterações significativas; USG doppler do sistema venoso do membro superior esquerdo com ausência de sinais de tombos venosa profunda; teste rápido para detecção de anticorpos anti-HIV 1 e 2 com resultado não reagente em duas amostras; hemocultura não reagente para as três amostras analisadas por duas ocasiões; LCR com variação dentro do intervalo de referência e sem alterações; e urocultura positiva para Klebsiella pneumoniae ssp pneumoniae

\section{CUIDADOS DE ENFERMAGEM}

A Enfermagem entendida como a arte do cuidar do sujeito-cidadão e de suas relações com o mundo em que inserido envolve todo o contexto biopsicossocial, cultural, espiritual e ecológico, desta forma, os profissionais estão engajados com o cuidar 
para com o usuário dos serviços de saúde, num processo de relações de cuidados entre humanos, uns com os outros e guarda íntima relação com as experiências de ser cuidado e de cuidar.

A arte de cuidar muito empregada na enfermagem pelo seu tato com pacientes, clientes e usuários dos serviços de saúde remete inevitavelmente a expressão empírica da atenção aos necessitados.

Elencamos desta maneira os cuidados que foram direcionados ao paciente em questão tratado neste relato: manutenção de dieta hipercalórica, monitoramento da oxigenação periférica, oferta de oxigênio suplementar se saturação periférica menor que $90 \%$, medição dos sinais vitais de quatro em quatro horas, mudança de decúbito de duas em duas horas, medição da diurese eliminada, realização de curativo conforme característica tecidual da úlcera por pressão em região sacra, vigilância no conciliar do sono, atenção aos sinais de hemorragia, infecção e fadiga, estímulo a orientação temporal e espacial, monitoramento dos sinais de abstinência alcoólica e promoção da hidratação corporal.

Os cuidados prestados pela equipe de enfermagem do setor de Tisiologia com a colaboração dos Residentes de Enfermagem em Pneumologia obteve como resultado a melhora substancial promovendo a alta hospitalar do paciente após três meses de internamento.
$\mathrm{Na}$ condição da alta hospitalar o cliente apresentava um padrão respiratório adequado, a úlcera por pressão apresentando tecido de granulação sem secreção ou odor diminuída a sua extensão, pele hidratada, padrão nutricional estabelecido, orientação espacial instituída, encaminhamento para Unidade de Saúde da Família.

\section{REFERÊNCIAS}

Ministério da Saúde (BR). [Página na Internet]. Brasília: Ministério da Saúde; Plano Nacional de Controle da Tuberculose/Plano Emergencial. [35] [acesso em 15 setembro 2002]. Disponível em:

http://www.saúde.gov.br/programas/Tubercul ose

Ministério da Saúde (BR). Manual técnico para o controle da Tuberculose: cadernos de atenção básica. $6^{\mathrm{a}}$ ed. Brasília (DF): MS; 2002.

Ministério da Saúde. Departamento de Vigilância Epidemiológica. Secretaria de Vigilância em Saúde. Programa Nacional de Controle da Tuberculose PNCT - Brasil. 2005

\section{ALBUQUERQUE MFM, LEITÃO CCS, CAMPELO ARL, SOUZAA WV, SALUSTIANO A. Fatores prognósticos para o desfecho da tuberculose pulmonar em Recife, Pernambuco, Brasil. Rev Panam Salud Publica, 2001, 9 (6); 368 - 74.}

Segatto ML, Pinsky I, Laranjeira R, Rezende FF, Vilela TR. Triagem e intervenção breve em pacientes alcoolizados atendidos na emergência: perspectivas e desafios. Cad Saúde Pública. jun 2007;23(8):1753-62. 
Andrade RLP, Villa TCS, Pillon SC. A influência do alcoolismo no prognóstico e tratamento da tuberculose. SMAD, Rev Eletrônica Saúde Mental Álcool Drog. (Ed. port.) [internet]. fev 2005;1(1). [acesso 12 abril 2014]. Disponível em:

http://www2.eerp.usp.br/resmad/verArtigo_po rt.php?idioma $=$ portugues $\&$ ano $=2005 \&$ volu $\mathrm{me}=1 \&$ numero $=1 \& \mathrm{id}=44$

SOUZA, S. S.; SILVA, D. M. G. V. (2010). Passando pela experiência do tratamento para tuberculose. Texto \& Contexto Enfermagem. Florianópolis, Out-Dez; 19(4): 636-643.

BERTAZONE, E. C.; GIR, E.;

HAYASHIDA, M. (2005). Situações vivenciadas pelos trabalhadores de enfermagem na assistência ao portador de tuberculose pulmonar. Revista Latinoamericana de Enfermagem, Maio-Junho; 13(3):374-81.

CHIRINOS, N. E. C.; MEIRELLES, B. H. S. (2011). Fatores associados ao abandono do tratamento da Tuberculose: uma revisão integrativa. Texto \& Contexto Enfermagem. Florianópolis, Jul-Set; 20(3): 599-406.

GALERA, S.A.F., and LUIS, M.A.V. "Principais conceitos da abordagem sistêmica em cuidados de enfermagem ao indivíduo e sua família." Revista da Escola de Enfermagem da USP 36.2 (2002): 141-147.

Brasil. Ministério da Saúde. Manual de recomendações para o controle da tuberculose no Brasil / Ministério da Saúde, Secretaria de Vigilância em Saúde, Departamento de Vigilância Epidemiológica. - Brasília : Ministério da Saúde, 2011.

BRASIL.III Diretrizes para Tuberculose da Sociedade Brasileira de Pneumologia e Tisiologia. J Bras Pneumol. 2009;35(10): 1018-1048.

BERTAZONE, DC, GIR, ÉE and HAYASHIDA, M. "Situações vivenciadas pelos trabalhadores de enfermagem na assistência ao portador de tuberculose pulmonar." Revista Latino-Americana de Enfermagem 13.3 (2005): 374-381.

ALMEIDA, AJF de, MONTENEGRO, HRA, and SANTOS, TCF. "A nova ordem no combate à tuberculose no Brasil: implicações para a enfermagem." Revista da Rede de Enfermagem do Nordeste-Rev Rene 10.1 (2012).

SILVA, RM, et al. "CUIDADOS DE ENFERMAGEM AO PACIENTE COM TUBERCULOSE PULMONAR." Revista Contexto \& Saúde 11.20 (2011): 859-864. 